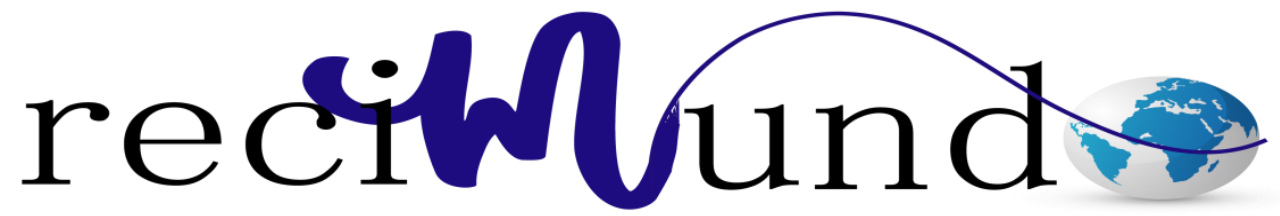

Revista Científica Mundo de la Investigación y el Conocimiento

José Eduardo Cedeño Gilces ${ }^{\mathrm{a}}$; Karen Brenda Sarmiento Segarra ${ }^{\mathrm{b}}$; Marco Alejandro Guerrero Casagualpa ${ }^{\mathrm{c}}$; Tamara Yadira Segovia Buitrón ${ }^{\mathrm{d}}$

Frecuencia de los tipos de prótesis total de cadera en pacientes intervenidos quirúrgicamente por coxartrosis en el hospital Guayaquil durante el periodo 20092011

Revista Científica Mundo de la Investigación y el Conocimiento. Vol. 2 núm., l, febrero, ISSN: 2588-073X, 2018, pp. 611-624

DOI: 10.26820/recimundo/2.1.2018.611-624

Editorial Saberes del Conocimiento

Recibido: 05/12/2017 Aceptado: 15/02/2018

a. Médico de la Universidad de Guayaquil; educedenomd@ @otmail.com

b. Médico de la Universidad de Guayaquil; karen_ue18@hotmail.com

c. Universidad Central de Ecuador; Facultad de Ciencias Médicas; Medico; marco88_08@ hotmail.com

d. Universidad Central de Ecuador Facultad de Ciencias Médicas; Médico; yadira17131317@gmail.com 


\section{Frecuencia de los tipos de prótesis total de cadera en pacientes intervenidos quirúrgicamente por coxartrosis en el hospital Guayaquil durante el periodo 2009-2011}

Vol. 2, núm. 1., (2018)

José Eduardo Cedeño Gilces; Karen Brenda Sarmiento Segarra; Marco Alejandro Guerrero Casagualpa; Tamara Yadira Segovia Buitrón

\section{RESUMEN}

La artroplastia total de cadera es una intervención quirúrgica comúnmente usada en la cual se reemplaza la articulación de la cadera por un implante protésico. Entre la indicación primaria de la artroplastia total de cadera es el alivio del dolor incapacitante en pacientes que no mejoran lo suficiente con medios no quirúrgico y para los que la única alternativa quirúrgica era la resección de la articulación. La cirugía proporciona éxito notable en pacientes con artrosis entre otras enfermedades.

El objetivo del estudio fue determinar la frecuencia de los tipos de prótesis total de cadera en pacientes intervenidos por artroplastia total de cadera con diagnóstico de coxartrosis en el Hospital de Especialidades Guayaquil “Dr. Abel Gilbert Pontón” durante el periodo 2009 al 2011.

El presente estudio es de tipo descriptivo, y retrospectivo, en el cual se revisaron 40 historias clínicas de los pacientes intervenidos quirúrgicamente en el Hospital Guayaquil.

El tipo de artroplastia total de cadera más frecuente fue NO CEMENTADA (80\%) de los casos. Como datos adicionales el grupo etario estuvo comprendido entre 20 y 97 años con un promedio de edad de 58,5 años, la distribución de los casos fue el $50 \%$ sexo masculino y el $50 \%$ sexo femenino.

Los cirujanos ortopédicos de un hospital deben cooperar con las autoridades del centro a fin de tomar criterios cabales para la selección de los implantes teniendo en cuenta las necesidades de cada paciente en la cual se determine estrategias para abarcar el coste del implante.

Palabras clave: Tipos de prótesis; cadera; artroplastia total. 


\section{Frecuencia de los tipos de prótesis total de cadera en pacientes intervenidos quirúrgicamente por coxartrosis en el hospital Guayaquil durante el periodo 2009-2011}

Vol. 2, núm. 1., (2018) José Eduardo Cedeño Gilces; Karen Brenda Sarmiento Segarra; Marco Alejandro Guerrero Casagualpa; Tamara Yadira Segovia Buitrón

\section{ABSTRACT}

Total hip arthroplasty is a commonly used surgical procedure in which replaces the hip joint by a prosthetic implant. Among the primary indication for total hip arthroplasty is disabling pain relief in patients who do not improve enough with nonsurgical means and for whom the only alternative was surgical resection of the joint. The surgery provides remarkable success in patients with osteoarthritis and other diseases.

The aim of the study was to determine the frequency of the types of total hip prosthesis in patients undergoing total hip arthroplasty with a diagnosis of osteoarthrosis in Guayaquil * Specialty Hospital Dr. Abel Gilbert Pontón “ during the period 2009 to 2011.

The present study is descriptive, and retrospective, in which we reviewed medical records of 40 patients operated on at the Hospital Guayaquil.

The type of total hip arthroplasty was NOT CEMENTED most frequent $(80 \%)$ cases. As additional data age group was between 20 and 97 years with a mean age of 58.5 years, the distribution of cases was $50 \%$ male and $50 \%$ female.

Orthopedic surgeons of a hospital should cooperate with the authorities to take center sane criteria for the selection of implants taking into account the needs of each patient determines which strategies to cover the cost of the implant.

Keywords: Types of prostheses; hip; total arthroplasty. 


\section{Frecuencia de los tipos de prótesis total de cadera en pacientes intervenidos quirúrgicamente por coxartrosis en el hospital Guayaquil durante el periodo 2009-2011}

Vol. 2, núm. 1., (2018)

José Eduardo Cedeño Gilces; Karen Brenda Sarmiento Segarra; Marco Alejandro Guerrero Casagualpa; Tamara Yadira Segovia Buitrón

\section{Introducción.}

La artrosis (OA) no puede ser descrita como una única enfermedad, sino como un grupo heterogéneo de patologías con manifestaciones clínicas similares, cambios patológicos y radiológicos comunes (1).

En los moradores españoles la frecuencia de la OA en algunas de las articulaciones es del 24\%. En los habitantes mayores de 20 años la frecuencia se considera del 10,2\% para la OA de rodilla y del 5,34\% para la OA de manos; si se examina los moradores superiores de 50 años la prevalencia de la OA de mano emerge hasta el 14\% (2).

La artrosis de cadera es una patología frecuente, imposiblitante, habitualmente se muestra en las dos articulaciones y moderadamente más usual en el sexo masculino así como otras formas de artrosis, su prevalencia asciende con la edad (3).

En el Ecuador alrededor del $70 \%$ de los habitantes reflejan radiológicamente la enfermedad pero los síntomas solo lo evidencian la mitad de ellos (4) .

La finalidad del tratamiento de la artrosis son: reducir la sintomatología, instruir al enfermo acerca de la artrosis, reducir la discapacidad y evitar o retrasar el florecimiento de la patología y sus resultados en el futuro (5).

La artroplastia total de cadera es una intervención quirúrgica comúnmente usada y se refiere al reemplazo de la articulación de la cadera por un implante protésico (6). 


\section{Frecuencia de los tipos de prótesis total de cadera en pacientes intervenidos quirúrgicamente por coxartrosis en el hospital Guayaquil durante el periodo 2009-2011}

Vol. 2, núm. 1., (2018)

José Eduardo Cedeño Gilces; Karen Brenda Sarmiento Segarra; Marco Alejandro Guerrero Casagualpa; Tamara Yadira Segovia Buitrón

Está conformado de 3 partes. La primera es el constituyente femoral la cual es un vástago que se encaja en el canal medular anticipadamente labrado (7). El segundo es el componente cefálico el cual es una esfera que se ensambla al vástago y por último el constituyente acetabular en donde se reemplaza la fracción de la articulación de la cadera, el mismo que es una esfera hueca que se adapta magníficamente al componente cefálico (7). Las prótesis de cadera pueden estar formadas de materiales como acero, polietileno, cemento (metilmetacrilato), cemento más antibiótico, cerámica, titanio entre otros (6).

El objetivo de la presente investigación es determinar la frecuencia de los tipos de prótesis total de cadera en pacientes intervenidos quirúrgicamente por coxartrosis en el Hospital Abel Gilbert Pontón periodo 2009-2011.

\section{Metodología.}

El presente estudio fue realizado en el área de estadística del Hospital Abel Gilbert Pontón ubicado en la ciudad de Guayaquil-Ecuador. El tiempo estimado de estudio comprende el periodo entre los años 2009 hasta el 2011; en este estudio se incluyen todos los pacientes con diagnóstico de coxartrosis que fueron intervenidos quirúrgicamente por ATC.

Los datos fueron incorporados a la correspondiente ficha de recolección, una por cada paciente, en las cuales se consignó el tipo de prótesis cementada, no cementada o hibrida así como su frecuencia. 


\section{Frecuencia de los tipos de prótesis total de cadera en pacientes intervenidos quirúrgicamente por coxartrosis en el hospital Guayaquil durante el periodo 2009-2011}

Vol. 2, núm. 1., (2018)

José Eduardo Cedeño Gilces; Karen Brenda Sarmiento Segarra; Marco Alejandro Guerrero Casagualpa; Tamara Yadira Segovia Buitrón

Se obtuvieron 90 pacientes diagnosticados con coxartrosis de los cuales 40 fueron intervenidos quirúrgicamente para ATC (44\%).

Tipo de investigación

Este estudio es Descriptivo, Retrospectivo.

Universo y muestra

Universo (Son todos los pacientes que fueron operados con diagnóstico de coxartrosis para colocación de prótesis total de cadera en el Hospital Dr. Abel Gilbert Pontón durante el periodo 2009-2011).

Técnicas de recolección de datos

Mediante la elaboración de formularios personalizados a cada una de las historias clínicas se recolecto toda la información necesaria en base a los requerimientos de los datos que se precisa obtener.

Procesamiento y análisis de datos

Los datos del formulario una vez obtenido fueron procesados en la hoja de datos Calc de open Office. 
Frecuencia de los tipos de prótesis total de cadera en pacientes intervenidos quirúrgicamente por coxartrosis en el hospital Guayaquil durante el periodo

2009-2011

Vol. 2, núm. 1., (2018)

José Eduardo Cedeño Gilces; Karen Brenda Sarmiento Segarra; Marco Alejandro Guerrero

Casagualpa; Tamara Yadira Segovia Buitrón

\section{Resultados.}

\begin{tabular}{|l|c|c|}
\hline \multicolumn{2}{|c|}{ TIPOS DE PROTESIS TOTAL DE CADERA } \\
\hline \multicolumn{1}{|c|}{ PROTESIS } & CASOS & $\%$ \\
\hline Cementada & 8 & 20 \\
\hline No Cementada & 32 & 80 \\
\hline Hibrida & 0 & 0 \\
\hline TOTAL & 40 & 100 \\
\hline
\end{tabular}

Tabla $N^{\circ}$ 1. Distribución de los tipos de prótesis de cadera

Prótesis total de cadera.

Se evaluaron retrospectivamente 40 casos de ATC en pacientes diagnosticados con osteoartrosis de cadera. En los cuales se evidencio los tipos de prótesis así como su frecuencia; el número de casos de las prótesis cementas fueron 8 , no cementadas 32 casos, no se observaron prótesis hibrida. De las prótesis no cementadas corresponden al $80 \%$ del total de los casos y las cementadas el restante $20 \%$. 
Frecuencia de los tipos de prótesis total de cadera en pacientes intervenidos quirúrgicamente por coxartrosis en el hospital Guayaquil durante el periodo 2009-2011

Vol. 2, núm. 1., (2018)

José Eduardo Cedeño Gilces; Karen Brenda Sarmiento Segarra; Marco Alejandro Guerrero Casagualpa; Tamara Yadira Segovia Buitrón

Pero adicionalmente se observó que el grupo etario presento edad promedio de 58.5 años, existiendo una prevalencia en pacientes de la quinta década con un $25 \%$ de casos, seguidos de pacientes en la cuarta década $20 \%$ de casos y posteriormente en sexta década $15 \%$ de los casos.

\begin{tabular}{|c|c|c|}
\hline EDAD & n & $\%$ \\
\hline $\mathbf{2 0 - 2 9}$ & 3 & 7 \\
\hline $\mathbf{3 0 - 3 9}$ & 5 & 12 \\
\hline $\mathbf{4 0 - 4 9}$ & 8 & 20 \\
\hline $\mathbf{5 0 - 5 9}$ & 10 & 25 \\
\hline $\mathbf{6 0 - 6 9}$ & 6 & 15 \\
\hline $\mathbf{7 0 - 7 9}$ & 4 & 10 \\
\hline $\mathbf{8 0 - 8 9}$ & 3 & 8 \\
\hline $\mathbf{9 0 - 9 9}$ & 1 & 3 \\
\hline TOTAL & 40 & 100 \\
\hline
\end{tabular}

Tabla $N^{\circ}$ 2. - distribución por grupo etáreo

Por otra parte se documenta según el género existieron igualdad en la serie de casos 50\%, con un total de 20 casos para el sexo masculino y 20 casos para el sexo femenino; de los cuales 1 caso corresponde a prótesis cementada y 19 a prótesis no cementada; 5\% prótesis cementada y 95\% prótesis no cementadas correspondientemente. En el sexo femenino se evidencio 7 casos de 


\section{Frecuencia de los tipos de prótesis total de cadera en pacientes intervenidos}

quirúrgicamente por coxartrosis en el hospital Guayaquil durante el periodo

2009-2011

Vol. 2, núm. 1., (2018)

José Eduardo Cedeño Gilces; Karen Brenda Sarmiento Segarra; Marco Alejandro Guerrero

Casagualpa; Tamara Yadira Segovia Buitrón

prótesis cementada que corresponde al 35\% y 13 casos para prótesis no cementada correspondiendo a $65 \%$. No existe evidencia de casos en pacientes con prótesis hibrida.

\begin{tabular}{|l|c|c|}
\hline & MASCULINO & FEMENINO \\
\hline PROTESIS CEMENTADA & 1 & 7 \\
\hline PROTESIS NO CEMENTADA & 19 & 13 \\
\hline TOTAL & 20 & 20 \\
\hline
\end{tabular}

Tabla $N^{\circ}$ 3.- Distribución por género

Pero adicionalmente se encontró complicaciones durante 3 años de estudio correspondiendo al 17\%. De las cuales el 57\% fueron luxaciones y el restante $43 \%$ fracturas.

Los casos de luxaciones fueron evidentes en pacientes con prótesis no cementada, en cambio las fracturas se observaron en prótesis no cementada 67\% (2 casos) y cementada $37 \%$ (1 caso). 
Frecuencia de los tipos de prótesis total de cadera en pacientes intervenidos quirúrgicamente por coxartrosis en el hospital Guayaquil durante el periodo 2009-2011

Vol. 2, núm. 1., (2018)

José Eduardo Cedeño Gilces; Karen Brenda Sarmiento Segarra; Marco Alejandro Guerrero Casagualpa; Tamara Yadira Segovia Buitrón

\begin{tabular}{|l|c|c|c|}
\hline & $\begin{array}{c}\text { PROTESIS } \\
\text { CEMENTADA }\end{array}$ & $\begin{array}{c}\text { PROTESIS } \\
\text { NO } \\
\text { CEMENTADA }\end{array}$ & $\begin{array}{c}\text { PROTESIS } \\
\text { HIBRIDA }\end{array}$ \\
\hline FRACTURAS & 1 & 2 & 0 \\
\hline LUXACION & 0 & 4 & 0 \\
\hline TOTAL & 1 & 6 & 0 \\
\hline
\end{tabular}

Tabla $N^{\circ}$ 4.- Tipos de complicaciones

\section{Discusión.}

El propósito de este estudio fue evaluar la frecuencia de los tipos de prótesis total de cadera que presentaron los 40 pacientes intervenidos quirúrgicamente con diagnosticados de coxartrosis. En nuestra serie, al analizar los resultados de los formularios, encontramos que el tipo de prótesis de cadera más utilizada fueron las no cementadas. Debemos de considerar que la mayoría de los pacientes que utilizaron prótesis no cementadas fueron pacientes menores de 60 años de edad, siendo estos más activos. La fijación cementada es satisfactoria para los pacientes ancianos con pocas demandas funcionales (7).

En otro estudio tenemos similitudes de datos, razonables con este estudio. Analizando los datos adicionales nos damos cuenta que se presentó igualdad de casos en ambos géneros, cosa contraria a lo que ocurre en varias revisiones. Alejandro Bermúdez en su estudio encontró 325 casos de ATC, con mayor prevalencia para las prótesis cementadas (9).

Los datos revelados de los tipos de prótesis demuestran que existió una elevada frecuencia de prótesis no cementadas $80 \%$, seguidas de prótesis cementadas $20 \%$. Fernando 


\section{Frecuencia de los tipos de prótesis total de cadera en pacientes intervenidos quirúrgicamente por coxartrosis en el hospital Guayaquil durante el periodo 2009-2011}

Vol. 2, núm. 1., (2018)

José Eduardo Cedeño Gilces; Karen Brenda Sarmiento Segarra; Marco Alejandro Guerrero

Casagualpa; Tamara Yadira Segovia Buitrón

Lopreite obtuvo resultados diferentes con una mayor frecuencia de prótesis hibridas $57.6 \%$, seguidos de prótesis cementadas $25.4 \%$ y el restante $17 \%$ prótesis no cementadas (18). Mark Pennington en la revista BMJ publicado en el 2013 demuestra mayor prevalencia de prótesis no cementadas $1,8 \%$, seguidas de prótesis hibridas $1.4 \%$ y posteriormente prótesis cementadas $1.2 \%$ (8). De igual manera Ali Abdulkarim en su estudio realizado en el año 2013 presento mayor frecuencia de prótesis no cementadas en un 51\% (13).

Adicionalmente se encontró según las edades mayor prevalencia de ATC en pacientes que se encuentran en la quinta década de su vida (25\%) de los casos, seguidos de pacientes con 40 años $(20 \%)$ y posteriormente pacientes de 60 años (15\%), resultados similares presento el estudio de Alfonzo Lugones (22). Sin embargo existen diferencias en dos estudios realizados por Fernando Lopreite en el cual la edad promedio en que se realizaron ATC fueron 64.4 años y 68 años respectivamente (11) (10).

Por otra parte existió igualdad de casos según la distribución del género, pero relacionando con otros estudios esto demuestra lo contrario, Fernando Lopreite presento mayor frecuencia en mujeres $61 \%$ de casos; de la misma manera Lie SA y colaboradores demuestra en su estudio publicado el año 2010 mayor prevalencia en el sexo masculino y mayores de 70 años (10) (23).

Pero como dato adicional las complicaciones presentadas en las ATC en este estudio corresponden al 17\%, de las cuales el 57\% fueron luxaciones y el restante $43 \%$ fracturas, el $67 \%$ se presentó en las prótesis no cementadas y el 37 \% restante en las prótesis cementadas. 


\section{Frecuencia de los tipos de prótesis total de cadera en pacientes intervenidos quirúrgicamente por coxartrosis en el hospital Guayaquil durante el periodo 2009-2011}

Vol. 2, núm. 1., (2018)

José Eduardo Cedeño Gilces; Karen Brenda Sarmiento Segarra; Marco Alejandro Guerrero Casagualpa; Tamara Yadira Segovia Buitrón

\section{Bibliografía.}

1. Zaragoza Brehcist, C. Patología osteoarticular en el anciano. Madrid: Medicine, 2006, Vol 9. 4029-4036.

2. Zhang, Yuqing. Epidemiologia la de la artrosis. Boston : Medicine, 2008, Vol. 1. 515529.

3. Blanco García. F. Artrosis. La Coruña : Medicine, 2005, Vol. 9. 2098-2107.

4. Santos Martín. J. Artrosis(2). Espondiloartrosis. Coxartrosis. Gonartrosis. Artrosis de manos. Otras localizaciones. Valladolid : Medicine, 2005, Vol. 9 . 2108-2116.

5. Moreira Palacios. F. Analisis Estadistico Exploratorio Para la Creacion de un Area de Reaumatología en un Hospital de la ciudad de Guayaquil. Guayaquil : Revista Tecnologica Espol, 2004. 1-179.

6. Ruiz, V. Artrosis de rodilla y cadera: diagnóstico clínico y papel de la radiología. [Fichas de consultas rapidas] Valencia : Sociedad Valencia de Medicina Familiar y Comunitaria, 2008. 1-4.

7. Sanz Sanz. J, Muñoz Carreño. P. Protocolo diagnóstico de la cadera dolorosa. Madrid : Medicine, 2005, Vol. 9. 2133-2136.

8. Reumatología, Sociedad Española de. Artrosis. fisiología, diagnóstico y tratamiento. Madrid : Editorial Medica Panamericana, 2010. 978-84-9835-330-3.

9. Pérez Díaz, M. Indicaciones de la resonancia magnética. Madrid : Medicine, 2007, Vol. 9. 1861-1864.

10. 10.Bernad Pineda, M. Actualización de Artrosis. Madrid : Grupo Saned, 2007. 1-49. 


\section{Frecuencia de los tipos de prótesis total de cadera en pacientes intervenidos quirúrgicamente por coxartrosis en el hospital Guayaquil durante el periodo 2009-2011}

Vol. 2, núm. 1., (2018) José Eduardo Cedeño Gilces; Karen Brenda Sarmiento Segarra; Marco Alejandro Guerrero Casagualpa; Tamara Yadira Segovia Buitrón

11. Ballina García. F, Alperi Lópeza. F. Manejo General y en medicina extrahospitalaria de la artrosis y enfermedades cristalinas. Oviedo : Medicine, 2009, Vol. 10. 2211-7.

12. Pablos Álvarez, J. Antiinflamatorios no esteroideos y analgésicos en las enfermedades articulares. Madrid : Medicine, 2005, Vol. 9. 2117-2123.

13. Duque Morán, J. Biomecánica de la prótesis total de cementada y no cementada. Barcelona : Canarias Médica y Quirúrgica , 2011, Vol. 9. 32-48.

14. Pennington, Mark. Protesis cementadas, no cementadas e hibridas para el reemplazo total de la cadera: analisis coste-eficacia . London : BMJ, 2013, Vol. 346. 1-14.

15. S, Terry Canales S,. Campbell Ciriugia Ortopedica. Madrid : Harcout Brace, 1998. 209518.

16. Lopreite, Fernando. El cementado y su posible relación con la infección aguda en las artroplastias totales de cadera.. Buenos Aires : Asoc Argent Ortop Traumatol, 2012, Vol. 77. $45-51$.

17. Bermúdez, Alejandro. Caracteristicas del reemplazo cadera en el Hospital Nacional Guillermo Almenara.. Lima : bvs, 1999, Vol. 59. 1025-5583.

18. Lopreite. Fernando;Garabano. G, Mana Pastrian. D, Robador. N \& Del Sel Hernan. Satisfacion del paceinte luego de una artroplastia de cadera o de rodilla.. Buenos Aires : Rev Asoc Argent Ortop Traumatol, 2012, Vol. 77. 112-117.

19. Abdulkarim, Ali. Cemented versus uncemented fixation in total hip replacement: a systematic review and meta-analysis of randomized controlled trials. Ireland : Pagepress Publicaciones, 2013, Vol. 5. 34-44. 
Frecuencia de los tipos de prótesis total de cadera en pacientes intervenidos quirúrgicamente por coxartrosis en el hospital Guayaquil durante el periodo 2009-2011

Vol. 2, núm. 1., (2018)

José Eduardo Cedeño Gilces; Karen Brenda Sarmiento Segarra; Marco Alejandro Guerrero Casagualpa; Tamara Yadira Segovia Buitrón

20. Alfonzo Lugones. G, Díaz. P. Artroplastia total de cadera con copa no cementada. Buenos Aires : Rev Asoc Argent Ortop Traumatol, 2012, Vol. 77. 185-191.

21. SA. Lie, Pratt. N. Duration of the increase in early postoperative mortality after elective hip. Bergen : JVJS, 2010, Vol. 92. 58-63. 